\title{
Pediatric Non-Infectious Osteomyelitis of the Mandible: A Case Report
}

\author{
Kyu-Hoon Lee', Seong-Yong Moon ${ }^{1}$, Jae-Seek You', Gyeong-Mi Kim', Nan-Young Lee ${ }^{2}$, Ji-Su Oh${ }^{1}$ \\ 'Department of Oral and Maxillofacial Surgery, School of Dentistry, Chosun University, Gwangju, Korea \\ ${ }^{2}$ Department of Pediatric Dentistry, School of Dentistry, Chosun University, Gwangju, Korea
}

Received March 27, 2020

Revised April 20, 2020

Accepted April 20, 2020

Correspondence to:

Ji-Su Oh

Department of Oral and Maxillofacial Surgery, School of Dentistry, Chosun

University, 309 Pilmun-daero, Dong-gu, Gwangju 61452, Korea

Tel: +82-62-220-3813

Fax: +82-62-222-3810

E-mail: jsoh@chosun.ac.kr

https://orcid.org/0000-0002-8369-5025

This study was supported by research funds from Education and Cultural Foundation of College of Dentistry, Chosun University, 2018.
Chronic recurrent multifocal osteomyelitis (CRMO) is a rare idiopathic inflammatory bone disease characterized by pain and swelling without any detectable infectious factors, the main feature is mild to moderate bone pain. CRMO commonly develops in the metaphyses of long bones and clavicles in children or adolescents. Chronic nonbacterial osteomyelitis (CNO) is the isolated form of CRMO and the etiology of CNO is still unclear. This report describes a rare case of CNO of the mandible in an 8-year-old female patient. On the basis of clinical, histological, and radiological findings, CNO was diagnosed. The patient was asymptomatic after surgical curettage followed by antibiotic therapy. Cone beam CT scan revealed a nearly completed bone healing after three months.

Key Words: Chronic recurrent multifocal osteomyelitis; Jaw diseases; Mandible; Osteomyelitis

\section{INTRODUCTION}

Chronic recurrent multifocal osteomyelitis (CRMO) is a rare multifocal inflammatory lesion of the bone without infection. CRMO was first reported by Giedion et al. [1] in 1972, it is a little-known disease. CRMO mainly occur in children and adolescents, and the median age of onset is 9 years old. CRMO affects predominantly females with female to male ratio at 2:1 [2]. The estimated prevalence is grossly 1-2 per million, it is considered as an orphan disease (www. orpha.net; OMIM \#259680) characterized by the recurrence of inflammatory bone pain [2,3].

Metaphyses and epiphyses of the long bones are most frequently affected, followed by the pelvis, clavicle, and vertebra. The involvement of mandible is very rare [4]. Nonspecific localized pain, tenderness, and swelling without infection can occur acute or chronic over 6 months and persistent or alternating resolve and recurrence [3].

CRMO can occur at any site of the bone, chronic nonbacterial osteomyelitis (CNO) is used to describe the isolated form of CRMO [2]. Girschick et al. [5] proposed that the descriptive name CNO might be more appropriate than CRMO because molecular or genetic diagnostic criteria are not available. Also, it is suggested CRMO should be used only in multifocal and recurrent cases [2,5].

CNO/CRMO is diagnosed through the clinical presentation, computed tomography (CT) or bone scan, bacterial culture, and bone biopsy. Since the CT does not show any specific evidence that distinguishes from bacterial osteomyelitis, bone biopsy and culture are essential for the definite and differential diagnosis [6]. Whereas infected bacterial osteomyelitis of the jaw is associated with odontogenic

Copyright (c) 2020 Korean Academy of Orofacial Pain and Oral Medicine. All rights reserved.

(c) This is an open-access article distributed under the terms of the Creative Commons Attribution Non-Commercial License (http://creativecommons.org/licenses/by-nc/4.0/), which permits unrestricted non-commercial use, distribution, and reproduction in any medium, provided the original work is properly cited. 
factors such as residual roots, periodontitis or dental caries, CNO/CRMO develops in the absence of infection [7]. Also, $\mathrm{CNO} / \mathrm{CRMO}$ occurs regardless of the radiation therapy or the administration of bisphosphonate.

The terminology of CNO/CRMO has been used inconsistently in the oral and maxillofacial surgery literature. The CNO/CRMO has been expressed as Garre's osteomyelitis, diffuse sclerosing osteomyelitis, primary chronic osteomyelitis, juvenile mandibular chronic osteomyelitis without a clear distinction from consistent terminology [3]. Especially, CNO/CRMO is poorly known in the field of dentistry because of a lack of information.

This report presents a rare pediatric case of unifocal noninfectious osteomyelitis with the painful osseous lesion in the mandible.

\section{CASE REPORT}

An 8-year-old girl was referred with a one month of history of pain and swelling of the right mandible. She had no previous history of trauma or any systemic disease. Physical examination revealed swelling and tenderness of the right mandibular angle area and posterior gingiva without trismus. There were no caries lesion or periodontal/periapical pathology as identifiable infectious sources on intraoral examination and panoramic radiograph (Fig. 1). Cone beam CT showed poorly defined osteolysis of the mandibular body with cortical perforation and outer cortical periosteal reaction (Fig. 2) Although no detectable infectious source was found, it was tentatively diagnosed as bacterial osteomyelitis based on cone beam CT findings. She was prescribed oral Augmentin 375 mg (amoxicillin-clavulanic acid) three times a day for 1.5 months without the administration of antibiotics, nonsteroidal anti-inflammatory drugs (NSAIDs). She underwent surgical curettage with bacterial culture. The inflammatory granulation tissue was attached to the periosteum and there was no pus formation. Also, the sources of dental origin to cause osteomyelitis were not observed. The histopathology demonstrated osteomyelitis with the presence of inflammatory fibrous tissue, microbiology was negative. Bone pain and swelling were significantly reduced within one month and had disappeared three months after surgical treatment. The newly formed bone was observed and the periosteal reaction was disappeared on cone beam CT (Fig. 3). She has not experienced any recurrence of symptoms or signs in the mandible during the follow-up period of 1 year.

\section{DISCUSSION}

CNO/CRMO is a sporadic inflammatory lesion of bone in children and adolescents. It is considered an

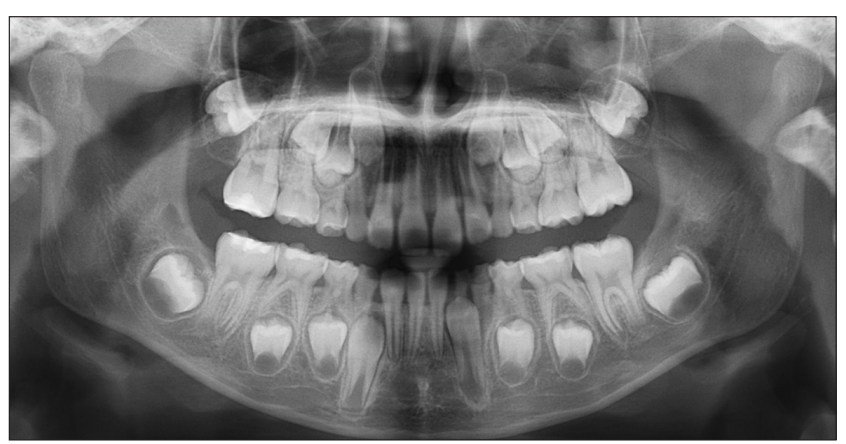

Fig. 1. Panoramic radiographic image of the patient at the first visit showed mixed dentition without any notable lesion.
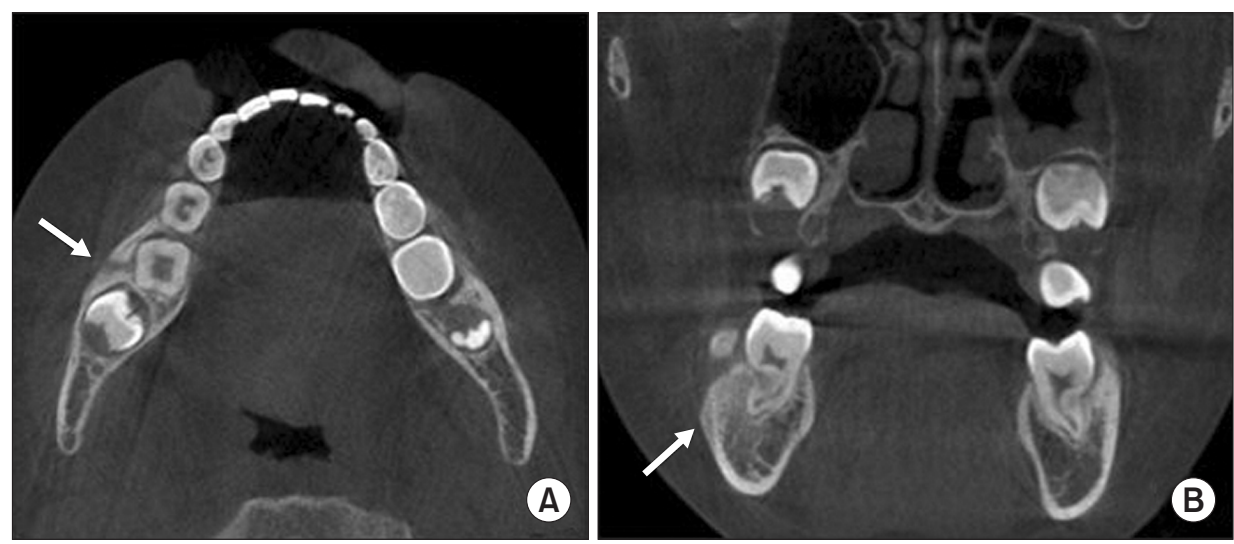

Fig. 2. The computed tomography scan confirms the (A) tunnel-like cortical lysis (arrow) and the (B) diffuse periosteal reaction (arrow) in the right mandibular body. 

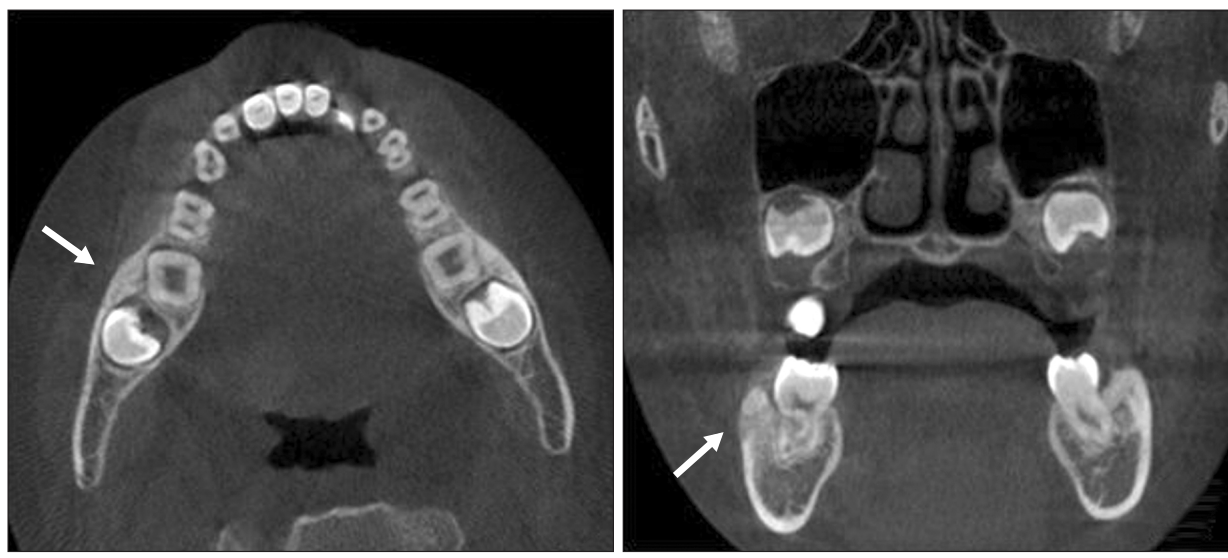

Fig. 3. A 3 months of follow-up computed tomography scan revealed the continuity of the cortical bone (arrows).

auto-inflammatory disorder rather than an autoimmune disease recently [8], the pathogenesis of CNO/CRMO is still controversial [2]. Although the hypothesis of the inflammation by a microorganism that could not be identified was accepted in the past $[9,10]$, polymerase chain reaction analysis was negative for microbial testing [5]. Various hypotheses such as immune deficiency, autoimmune response triggered by a micro-organism, immune barrier breakdown caused by normal skin antigens have been proposed, the exact pathogenetic mechanism has not been clearly identified [6].

The mandibular CNO is estimated to be approximately $2 \%$ of the CRMO (Fig. 4) [4,5]. The mandible is the most commonly affected bone as unifocal osteomyelitis [3]. In 73\% of patients, the jaw was the first manifestation of the inflammatory disease [3], this may suggest that the possibility of development of CRMO.

Clinical features of CNO/CRMO include the following symptoms: pain and swelling of the affected lesion, trismus, and headache [7]. Otalgia, warmth, erythema, rare pathologic fracture also can be found in some cases [3]. The levels of inflammatory factors such as white blood cells, C-reactive protein (CRP), and erythrocyte sedimentation rate may be in the normal range or may increase slightly [7].

Diagnosis is based on clinical, radiological and histological evaluations. There are many diagnostic criteria for $\mathrm{CNO} /$ CRMO. The latest diagnostic criteria according to Roderick are as follows [8]: 1) the presence of typical clinical features: bone pain and/or localized swelling without features of local or systemic infection, 2) radiological findings: osteolysis of the cortical bone with sclerosis of medullary space

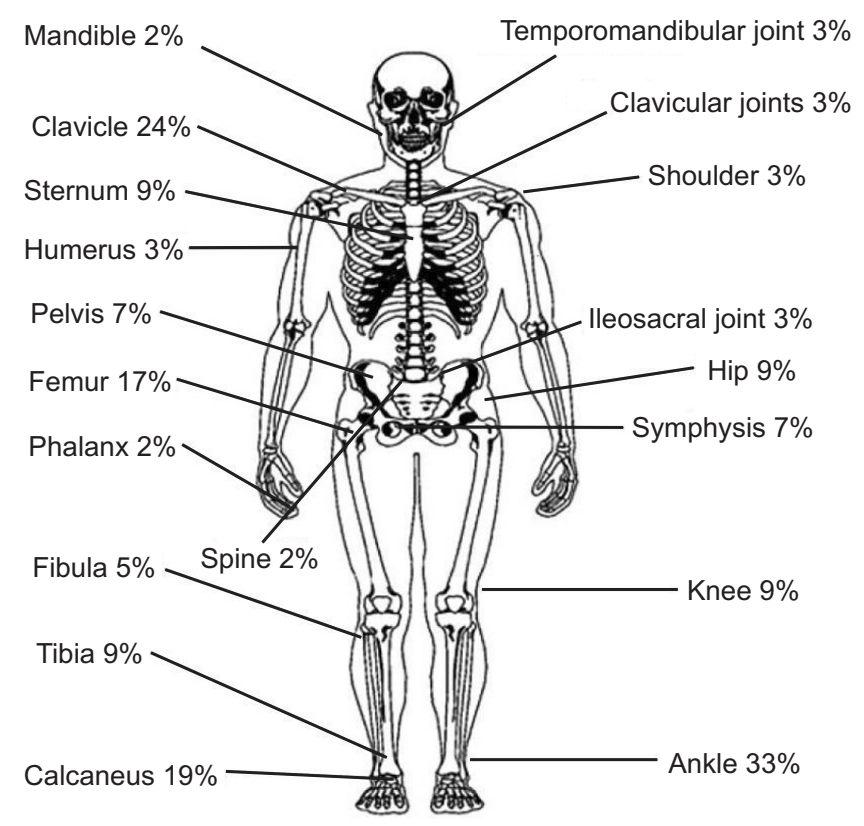

Fig. 4. The percentages of affected bones or joints are shown in pediatric patients with chronic nonbacterial osteomyelitis. Reprinted from the article of Girschick et al. [5] (Ann Rheum Dis 2005;64:279285) with original copyright holder's permission.

and periosteal reaction, and either of 1) more than one bone without significantly raised CRP under $30 \mathrm{~g} / \mathrm{L}, 2)$ if unifocal, or more than $30 \mathrm{~g} / \mathrm{L}$ of CRP with inflammatory changes in the bone biopsy with no bacterial growth whilst not on antibiotic therapy.

In cone beam CT image, the early stage of CNO of the mandible shows extensive resorption of the cortical and trabecular bone with cortical destruction. In the chronic stage, a mixed form of sclerotic changes and osteolytic changes can be found [3,6]. Radiographic findings include various types such as osteolysis, osteitis, hyperostosis, and 
osteosclerosis depending on the stage of the disease [11].

Differential diagnosis of CNO/CRMO in the mandible must include infectious osteomyelitis, osteosarcoma, Ewing sarcoma, fibrous dysplasia, Langerhans cell histiocytosis, and other diseases. The clinical and radiological characteristics of CNO/CRMO are very similar to infectious osteomyelitis, infectious osteomyelitis can be excluded because $\mathrm{CNO} /$ CRMO show features of subacute or chronic culture-negative osteomyelitis [9,11]. CNO/CRMO can be misdiagnosed as fibrous dysplasia because of the irregular radiopacify on panoramic radiographs. CT is helpful to exclude fibrous dysplasia because the periosteal reaction is not seen in fibrous dysplasia [3]. The pattern of the infiltrative resorption of the cortical and trabecular bone in the early stage is similar malignant bone disease such as osteosarcoma or Ewing's sarcoma, it is important to undertake the biopsy of cortical and cancellous bone. Some surgeons recommend an extraoral approach for biopsy to avoid oral contamination. The smooth and lamellated appearance of periosteal new bone formation in CT helps to differentiate CNO/CRMO from neoplastic diseases [3,6].

The purpose of treatment is reducing pain and control of the inflammation which prevents the destruction of bone and progression of the disease. Although there is no definitive treatment for CNO/CRMO [11], the conservative therapy with the administration of NSAIDs, and short-term corticosteroids, immune modulators, calcitonin, antimetabolites, and colchicine, either alone or in combination has been suggested [11,12]. Rasmussen et al. [13] reported that antibiotic therapy for several months was not effective with no clinical response.

NSAIDs such as naproxen, indomethacin is recommended as the first choice for the initial treatment currently $[2,14]$. NSAIDs are most likely to alter the course of the disease because they control the pain in most cases and prostaglandins play an important role in osteoclast activation and bone remodeling [15]. Despite the use of NSAIDs, if the pain is unresolved or recurrent, intensive anti-inflammatory treatment using oral corticosteroids, anti-tumor necrosis factor agents, methotrexate, and bisphosphonates is an alternative [16]. Bisphosphonate as the second choice relieves pain by inhibiting the synthesis of pro- and anti-inflammatory cytokines such as TNF- $\alpha$, IL- 6 , and IL-1 [17]. CNO/
CRMO is expansile with osteoclast-rich edge, inhibition of osteoclast by bisphosphonate reduce the expansion of $\mathrm{CNO} /$ CRMO [18]. Especially, bisphosphonate is effective in cases of bone remodeling accompanied by sclerosis [2]. Although pamidronate is an effective second choice therapy, close monitoring is essential because it has potential risk such as irreversible osteopetrosis and long-term data is lacking in pediatric patients. Further evaluation of long-term efficacy and safety of bisphosphonate is needed [16]. The surgical approach may be needed for persistent cases although it is not the first choice for the treatment of CNO.

It has been reported that the CNO persisted despite the prolonged administration of antibiotics for several months [13]. Fortunately, surgical treatment with administration of antibiotic was effective without the administration of NSAIDs, corticosteroid or bisphosphonate in this case. Maybe this case is thought to have been found relatively early phase. CNO/CRMO is easy to misdiagnose like this case because it is not a well-known disease and it has similar clinical characteristics to other infectious diseases, especially bacterial osteomyelitis.

In a cohort study of Wipff et al. [4], no remission, patients who needed therapy with anti- TNF- $\alpha$ and/or bisphosphonate, male, multifocal osteomyelitis at the onset, and a long period of time between onset and diagnosis present poor prognosis.

It is unclear whether CNO in the mandible is a unifocal disease or as one of the lesions CRMO [3]. CNO in the mandible may suggest CRMO including multiple osteomyelitis and/or extraosseous inflammatory disease. Although CNO/ CRMO is considered a benign disease, periodic long-term follow-up is important because it is characterized by alternating relief and recurrence. Although CNO/CRMO occurs very rarely in the oral and maxillofacial field, the exclusion for CNO/CRMO is essential in cases of osteomyelitis which cannot be found infectious factors in children and adolescents.

\section{CONFLICT OF INTEREST}

No potential conflict of interest relevant to this article was reported. 


\section{ORCID}

\author{
Kyu-Hoon Lee \\ https://orcid.org/0000-0002-0236-1788 \\ Seong-Yong Moon \\ https://orcid.org/0000-0002-7513-4404 \\ Jae-Seek You \\ https://orcid.org/0000-0001-7638-9583 \\ Gyeong-Mi Kim \\ https://orcid.org/0000-0002-0451-379X \\ Nan-Young Lee \\ https://orcid.org/0000-0002-4738-9389 \\ Ji-Su Oh \\ https://orcid.org/0000-0002-8369-5025
}

\section{REFERENCES}

1. Giedion A, Holthusen W, Masel LF, Vischer D. [Subacute and chronic "symmetrical" osteomyelitis]. Ann Radiol (Paris) 1972;15:329-342.

2. Chen Z, Cheng L, Feng G. Bone inflammation and chronic recurrent multifocal osteomyelitis. Eur Rev Med Pharmacol Sci 2018;22:1380-1386.

3. Padwa BL, Dentino K, Robson CD, Woo SB, Kurek K, Resnick CM. Pediatric chronic nonbacterial osteomyelitis of the jaw: clinical, radiographic, and histopathologic features. J Oral Maxillofac Surg 2016;74:2393-2402.

4. Wipff J, Costantino F, Lemelle I, et al. A large national cohort of French patients with chronic recurrent multifocal osteitis. Arthritis Rheumatol 2015;67:1128-1137.

5. Girschick HJ, Raab P, Surbaum S, et al. Chronic non-bacterial osteomyelitis in children. Ann Rheum Dis 2005;64:279-285.

6. Monsour PA, Dalton JB. Chronic recurrent multifocal osteomyelitis involving the mandible: case reports and review of the literature. Dentomaxillofac Radiol 2010;39:184-190.
7. Kim SM, Lee SK. Chronic non-bacterial osteomyelitis in the jaw. J Korean Assoc Oral Maxillofac Surg 2019;45:68-75.

8. Roderick MR, Shah R, Rogers V, Finn A, Ramanan AV. Chronic recurrent multifocal osteomyelitis (CRMO) - advancing the diagnosis. Pediatr Rheumatol Online J 2016;14:47.

9. Girschick HJ, Huppertz HI, Harmsen D, Krauspe R, MüllerHermelink HK, Papadopoulos T. Chronic recurrent multifocal osteomyelitis in children: diagnostic value of histopathology and microbial testing. Hum Pathol 1999;30:59-65.

10. Hummell DS, Anderson SJ, Wright PF, Cassell GH, Waites KB. Chronic recurrent multifocal osteomyelitis: are mycoplasmas involved? N Engl J Med 1987;317:510-511.

11. Gleeson H, Wiltshire E, Briody J, et al. Childhood chronic recurrent multifocal osteomyelitis: pamidronate therapy decreases pain and improves vertebral shape. J Rheumatol 2008;35:707-712.

12. Borzutzky A, Stern S, Reiff A, et al. Pediatric chronic nonbacterial osteomyelitis. Pediatrics 2012;130:e1190-e1197.

13. Rasmussen AQ, Andersen UB, Jørgensen NR, Schwarz P. Noninfectious osteomyelitis of the mandible in a young woman: a case report. J Med Case Rep 2014;8:44.

14. Job-Deslandre C, Krebs S, Kahan A. Chronic recurrent multifocal osteomyelitis: five-year outcomes in 14 pediatric cases. Joint Bone Spine 2001;68:245-251.

15. Hedrich CM, Hofmann SR, Pablik J, Morbach H, Girschick HJ. Autoinflammatory bone disorders with special focus on chronic recurrent multifocal osteomyelitis (CRMO). Pediatr Rheumatol Online J 2013;11:47.

16. Compeyrot-Lacassagne S, Rosenberg AM, Babyn P, Laxer RM. Pamidronate treatment of chronic noninfectious inflammatory lesions of the mandible in children. J Rheumatol 2007;34:15851589.

17. Miettunen PM, Wei X, Kaura D, Reslan WA, Aguirre AN, Kellner JD. Dramatic pain relief and resolution of bone inflammation following pamidronate in 9 pediatric patients with persistent chronic recurrent multifocal osteomyelitis (CRMO). Pediatr Rheumatol Online J 2009;7:2.

18. Simm PJ, Allen RC, Zacharin MR. Bisphosphonate treatment in chronic recurrent multifocal osteomyelitis. J Pediatr 2008;152:571-575. 